\title{
Reconstrução estético-funcional das pálpebras superior e inferior em paciente pediátrico após ataque canino: relato de caso
}

Laiz Moreira de PAULA ${ }^{1}$; Agnaldo Rocha PRATA JÚNIOR'; Maiolino Thomaz Fonseca OLIVEIRA ${ }^{2}$

1 - Residente em Cirurgia e Traumatologia Bucomaxilofacial do Hospital Estadual de Urgências Governador Otávio Lage de Siqueira (HUGOL), Goiânia - GO, Brasil; 2 - Cirurgião Bucomaxilofacial do Hospital Estadual de Urgências Governador Otávio Lage de Siqueira (HUGOL), Goiânia - GO, Brasil.

\section{Resumo}

Ataques caninos podem causar lesões graves e desconfigurantes na face de crianças, ao ponto de necessitarem de atendimentos especializados e de urgência. O acometimento da região periocular requer uma avaliação completa para identificar possíveis fraturas ósseas, lesões palpebrais, oculares e no sistema lacrimal, juntamente com o acompanhamento oftalmológico. O objetivo desse artigo é relatar o caso de uma criança de 07 anos de idade, vítima de mordedura por cão em face. $\mathrm{O}$ ataque resultou na avulsão parcial das pálpebras superior e inferior do olho direito. Após realizado o procedimento de reconstrução palpebral e dos cuidados profiláticos antirrábicos, o paciente evolui com satisfatório reestabelecimento estético-funcional e sem sinais e sintomas da doença raiva. Esse relato enfatiza a importância do manejo adequado frente aos ferimentos causados por ataque canino na região palpebral, em um paciente pediátrico.

PALAVRAS-CHAVE: Mordeduras e picadas; Cães; Pálpebras; Cirurgiões Bucomaxilofaciais; Ferimentos e lesões.

AUTOR PARA CORRESPONDÊNCIA

Laiz Moreira de Paula

Rua T-33, n. 125, Like Bueno Residence, Setor Bueno, Goiânia - G0, Brasil.

Telefone: +55 (62) 9 9822-9675

E-mail: laizmoreirap@gmail.com 


\section{Introdução}

As mordeduras causadas por animais são lesões potencialmente graves e desfigurantes, comumente vistas nos serviços de urgência e emergência. Dentre os casos notificados, os cães são os animais mais responsáveis por esses ataques e a maior parte dos pacientes acometidos são pediátricos ${ }^{1}$. As feridas causadas por mordedura animal são potencialmente contaminadas e necessitam de tratamento especializado, devido ao alto risco de infecção e complicações associadas².

Quando o trauma está relacionado à face, os relatos literários são raros e essa região prevalece entre os pacientes pediátricos ${ }^{3}$. Nesses casos, é indicada a realização de um minucioso exame para identificar possíveis fraturas ósseas, lesões cerebrais, hemorragias intensas e o grau de acometimento funcional das estruturas faciais ${ }^{4,5}$. A região periocular, quando acometida, pode causar lesões graves e um resultado insatisfatório devido à alta complexidade anatômica das pálpebras ${ }^{6}$.

A reconstrução palpebral tem como desafio reestabelecer a função e a delicada estética que é fornecida pelas camadas lamelares e músculos perioculares. A definição correta do tratamento visa prevenir complicações como ptose, ectrópio, entrópio, lagoftalmo e retração palpebral ${ }^{7}$. O presente relato de caso tem como objetivo apresentar o tratamento proposto para reconstrução das pálpebras superior e inferior direitas de um paciente pediátrico, após ataque por cão.

\section{Relato do caso}

Paciente do sexo masculino, 07 anos de idade, morador de zona rural, foi admitido em um hospital de urgências após ser vítima de mordedura por cão em face. Na admissão, o paciente encontrava-se em bom estado geral e segundo acompanhante, o mesmo não possuía comorbidades nem alergias medicamentosas. $\mathrm{O}$ cachorro era familiar, não era vacinado contra raiva e foi sacrificado pela família, logo após o acidente. 
A criança foi acometida somente na região da face. Fisicamente, apresentava múltiplos ferimentos puntiformes em hemiface direita, ferimento cortocontuso em lábio superior e avulsão parcial das pálpebras superior e inferior do olho direito. A pálpebra superior foi seccionada em espessura total na região da prega supratarsal, já a pálpebra inferior foi seccionada ao longo da margem palpebral e da região pré-tarsal. Não houve perda de substância tecidual e os tecidos deslocados se mostravam pediculados e viáveis. Os cantos medial e lateral estavam intactos, não houve traumatismo do sistema lacrimal e do bulbo ocular e assim como, não houve fraturas de ossos faciais. A acuidade visual, motilidade ocular e reatividade pupilar direta e consensual estavam preservadas em ambos os olhos (Figura 1).

O paciente foi imediatamente encaminhado ao centro cirúrgico em caráter de urgência para tratamento das lesões traumáticas pela equipe de Cirurgia e Traumatologia Bucomaxilofacial do Hospital Estadual de Urgências Governador Otávio Lage de Siqueira. Ele foi submetido ao procedimento de reconstrução das pálpebras superior e inferior do olho direito e sutura dos múltiplos ferimentos sob sedação endovenosa, com uso de dexmedetomidina, cetamina e propofol.

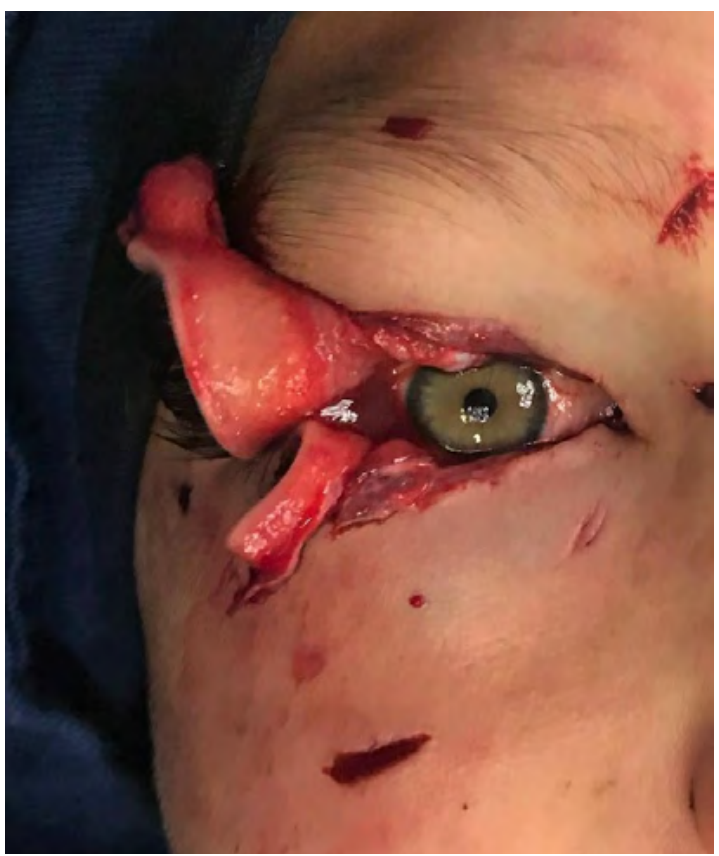

FIGURA 1 - Trauma periocular envolvendo a avulsão parcial das pálpebras superior e inferior do olho direito 
Ao primeiro passo, foi realizada infiltração anestésica local com lidocaína 2\% e epinefrina 1:200.000 diluída em água destilada, limpeza copiosa dos ferimentos com soro fisiológico e hemostasia com compressão suave e eletrocautério. O músculo orbicular do olho foi dissecado das bordas do tarso para permitir o fechamento primário. As pálpebras foram gentilmente manuseadas com pinça micro dente de rato até o encontro minucioso das margens interrompidas. $\mathrm{O}$ alinhamento correto da pálpebra foi verificado pela simetria entre os olhos. A aproximação dos tecidos profundos foi realizada com suturas interrompidas de espessura parcial e com os nós para anterior, utilizando fio absorvível Vicryl 5-0. Os defeitos da margem palpebral e da pele foram suturados com pontos interrompidos com Nylon 6-0 (Figura 2). Cuidados foram tomados para que o bulbo ocular não fosse lesionado durante o procedimento, através do uso de afastadores e espátula maleável metálica.

Foi realizada antibioticoterapia com Amoxicilina e Clavulanato de potássio $(75 \mathrm{mg} / \mathrm{kg} / \mathrm{dia})$, soro antirrábico e esquema de 04 doses da vacina. A ocorrência foi notificada ao Conselho Tutelar

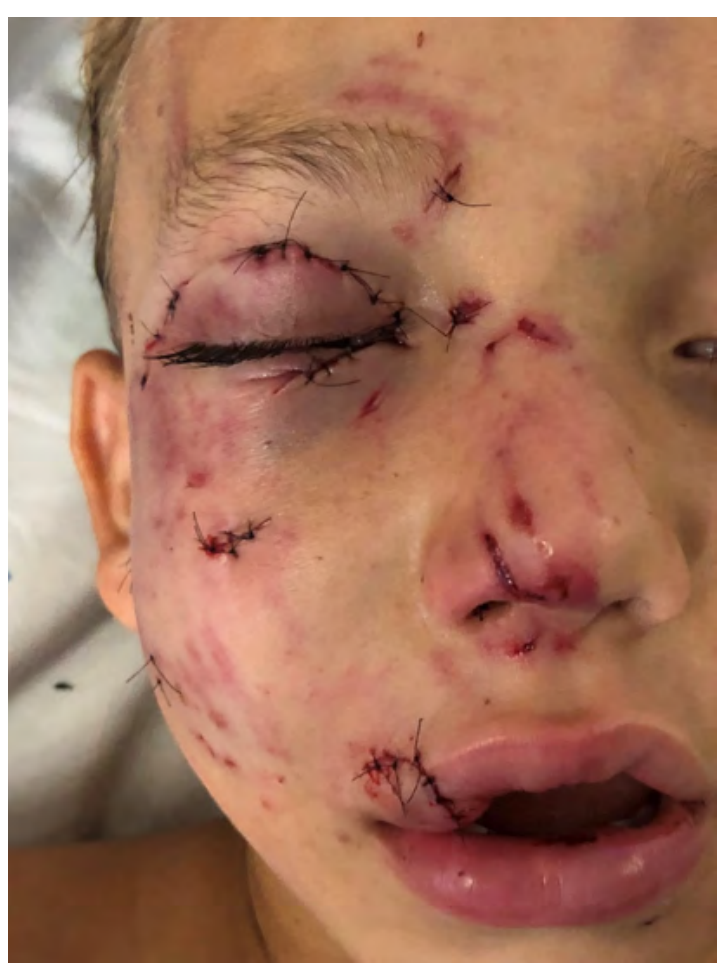

FIGURA 2 - Pós-operatório imediato da reconstrução dos ferimentos em hemiface direita 
da cidade. O paciente foi encaminhado para exame oftalmológico durante o período de internação, em que se pode observar movimentação ocular externa preservada, olho calmo, câmera anterior formada no olho direito e foi sugerida a manutenção da antibioticoterapia profilática e uso de prednisolona endovenosas.

As suturas foram removidas após 07 dias e o paciente fez uso de laserterapia de baixa potência para acelerar a reparação tecidual. Foi utilizado o laser vermelho em dose de 1 Joule nas pálpebras direitas, sem o uso de óculos de proteção, mantendo o fechamento das pálpebras superior e inferior, e em dose de 3 Joules nos outros ferimentos, com o uso de óculos de proteção. O paciente foi acompanhado periodicamente durante 10 meses. Os ferimentos evoluíram com bom aspecto de cicatrização e não foram observadas sequelas estético-funcionais. Não foram necessários procedimentos cirúrgicos reparadores adicionais (Figura 3).

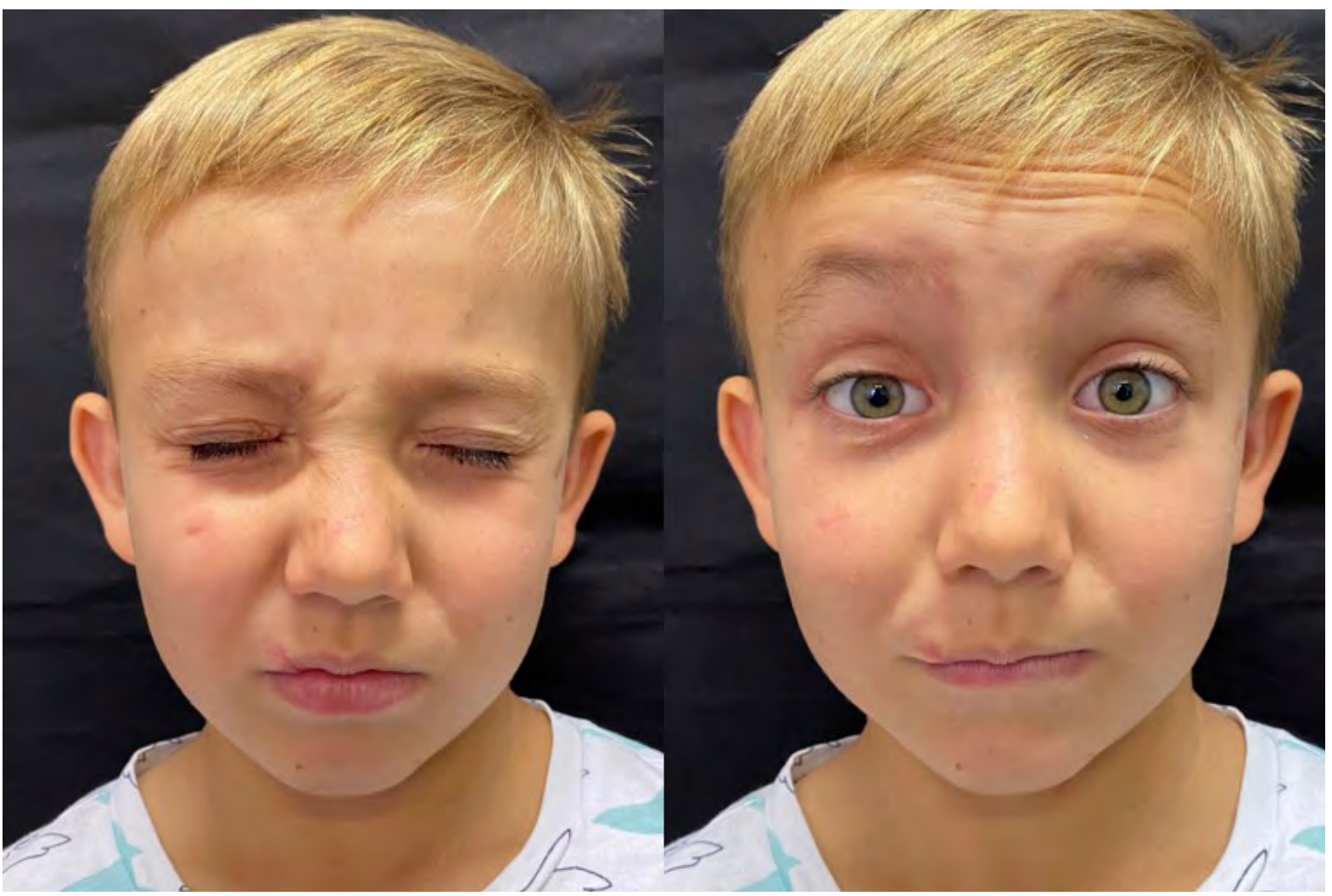

FIGURA 3 - Pós-operatório de 10 meses evidenciando o reestabelecimento da função e da estética das pálpebras superior e inferior do olho direito 


\section{Discussão}

Os animais domésticos, como cães e gatos, comumente atacam crianças na região de cabeça e face devido a maior proporção do tamanho da cabeça em relação ao corpo, por ser uma região de maior exposição, pela dificuldade das crianças em realizar respostas de defesa e reflexo e pela necessidade de trazer o rosto próximo ao animal ${ }^{3,8,10}$. Na maioria dos casos, o cão agressor é conhecido ou da própria família e deve ser observado rigorosamente para avaliar se possui sinais da raiva, uma doença infecciosa 9 .

Nos estudos e casos clínicos que abordam crianças vítimas de ataque animal em face, o local prevalente foi a região de terço médio ${ }^{8,10}$. O paciente do presente caso apresentado foi acometido com ferimentos puntiformes na região de terço médio, mas a lesão de maior gravidade ocorreu na região periocular. Devido à extensão da lesão, proximidade com estruturas nobres, necessidade de abordagem imediata e trauma psicológico causado na criança, a intervenção em centro cirúrgico com caráter de urgência foi necessária.

As lesões provocadas no bulbo ocular são relativamente raras. $\mathrm{Na}$ região periocular, os traumas resultam, mais frequentemente, em lesões das pálpebras e do sistema lacrimal, e mesmo assim, devem ser avaliados pelo médico oftalmologista o quanto antes ${ }^{3,7}$. Em muitos casos, esse profissional especialista não está presente nos hospitais de urgência para a avaliação inicial, e o acompanhamento deve seguir após a primeira intervenção. Os traumas pediátricos são desafiadores devido à dificuldade em realizar avaliação primária, resposta fisiológica diferente da do adulto, características anatômicas únicas, necessitando de decisões rápidas e acompanhamento em conjunto pela equipe de pediatria.

A alta complexidade da reconstrução das pálpebras exige uma avaliação completa do tamanho, formato e local do defeito e se há tecido sadio para reconstrução direta, para definição do melhor procedimento a ser realizado ${ }^{6,11}$. As possibilidades de tratamento dos ferimentos incluem o fechamento direto, reparação 
por segunda intenção ou utilização de retalhos e enxertos. Após a aproximação dos tecidos traumatizados, foi concluído que se tratava de defeitos palpebrais com perda menor de $25 \%$ de largura, sendo altamente indicado o fechamento primário ${ }^{7,11-13}$. As pálpebras dos pacientes pediátricos são firmes, carecem de rugas e linhas de expressão, portanto exigem maior refinamento da técnica cirúrgica para obtenção de um resultado estético favorável.

O reestabelecimento anatômico das palpébras visou devolver os mecanismos de fechamento, contorno do globo pela margem palpebral, lubrificação do globo e proteção à visão. Para isso, as pálpebras foram fechadas em duas camadas distintas: lamela anterior e lamela posterior. A camada anterior é composta pela pele e pelo músculo orbicular do olho, e a posterior, pela placa tarsal e a conjuntiva ${ }^{14,15}$. A camada posterior não deve ferir a córnea e por esse motivo, as suturas não podem ser dispostas em espessura total ${ }^{16}$.

A reconstrução da pálpebra superior exige um maior cuidado técnico devido ao risco de ptose e lagoftalmo ${ }^{17}$. Por realizar movimentos de abertura e fechamento, a pálpebra superior deve ser reconstruída de forma que seja mantida sua flexibilidade ${ }^{13,18}$. O reestabelecimento da prega supratarsal possibilitou a reinserção do músculo elevador superior da pálpebra superior. Já o ferimento da pálpebra inferior envolveu a região pré-tarsal e a margem palpebral, regiões que exigem um fechamento cauteloso. Deve-se cuidadosamente alinhar a linha cinza e linha dos cílios e evitar tensão horizontal excessiva ${ }^{11,12}$. Diferentemente da pálpebra superior, a reconstrução da pálpebra inferior requer apenas manutenção da estabilidade ${ }^{19}$.

Raramente são encontrados defeitos que envolvem ambas as pálpebras, o que aumenta ainda mais a complexidade de resolução do caso. Os princípios permanecem os mesmos que para reconstrução isolada da pálpebra ${ }^{20}$. O manuseio das pálpebras deve ser realizado com cuidado para evitar a perda do tecido viável. As mesmas devem ser descoladas e aparadas para evitar exposição das bordas do tarso ${ }^{16}$. Nesse caso, não foi necessário 
realizar as técnicas de cantólise e cantotomia lateral para possibilitar o fechamento primário, nem a utilização de enxertos, pois o tecido avulsionado estava em boas condições e foi suficiente para a reconstrução.

Durante muitos anos, a recomendação era que os ferimentos de mordedura permanecessem abertos para cicatrização por segunda intenção para evitar possíveis infecções, devido ao alto índice de contaminação e infecção ${ }^{21}$. A literatura atual preconiza o fechamento imediato dessas lesões para maximizar o ganho estético e funcional ${ }^{8,10,22}$. Irrigação abundante com solução salina, fechamento correto, profilaxia antibiótica, cuidados para evitar a infecção pela raiva e acompanhamento rigoroso são os princípios requisitados para obter sucesso no tratamento dos ferimentos causados por mordeduras animais ${ }^{6,9}$.

As complicações encontradas após reconstrução palpebral são relativamente comuns e incluem: deiscência de sutura, hemorragias, ectrópio, ptose, entrópio, retração palpebral, perda de suporte da pálpebra ${ }^{7,16}$. O uso do laser de baixa potência foi utilizado com o objetivo de acelerar o processo de cicatrização tecidual e evitar as complicações pós-operatórias ${ }^{23}$, mantendo os cuidados para não direcionar o feixe diretamente ao bulbo ocular através do fechamento das pálpebras e em uma dose menor. O paciente do caso relatado não apresentou nenhuma complicação, o que comprova que as mesmas podem ser evitadas por meio de uma avaliação detalhada, correta indicação da técnica e cumprimento dos cuidados necessários ${ }^{24}$.

Como preconizado pelo Ministério da Saúde ${ }^{25}$, foram realizadas as 04 doses da vacina antirrábica e 01 dose do soro antirrábico como medida profilática pós-exposição para raiva, já que o animal agressor morreu e não poderia ser observado. $O$ antibiótico mais indicado para terapia profilática é a Amoxicilina associada ao ácido clavulânico ou a Cefalexina ${ }^{8-10}$. Os tecidos perioculares são ricamente irrigados, por isso raramente é observado necrose, inviabilidade do tecido lacerado e relato de infecções ${ }^{9,11,21}$. 
Por isso, ainda é controverso e necessário mais estudos sobre a real necessidade da utilização de profilaxia antibiótica em mordeduras caninas em face. Nesse caso, antibiótico profilático de escolha foi a amoxicilina associada ao ácido clavulânico e o paciente não apresentou processo infeccioso nos ferimentos.

\section{Conclusões}

Apesar do acidente com animal doméstico ser uma situação totalmente evitável, ainda é considerado um problema social grave e que causa severas deformidades aos pacientes pediátricos. A correta avaliação e definição da terapia, associadas ao conhecimento da delicada anatomia palpebral e das opções de reparo dos defeitos, geralmente resulta em uma excelente função palpebral, sobrevida ocular e aparência estética, com mínimas complicações.

\section{Referências}

1 - Wladis EJ, Dewan MA. Periorbital trauma from pit bull terrier attacks. Orbit. 2012; 31(3): 200-2. doi: 10.3109/01676830.2012.669008.

2 - Ward MA. Bite Wound Infections. Clin Pediatr Emerg Med. 2013; 14(2): 88-94. doi 10.1016/j.cpem.2013.04.006.

3 - Bratton EM, Golas L, Wei LA, Davies BW, Durairaj VD. Ophthalmic Manifestations of Facial Dog Bites in Children. Ophthalmic Plast Reconstr Surg. 2018; 34(2): 106-109. doi: 10.1097/IOP.0000000000000875.

4 - Raj A, Thakur S, Arya KS, Kesarwani P, Sinha U. Canalicular lacerations in a tertiary eye hospital: our experience with monocanalicular stents. Rom J Ophthalmol. 2020; 64(2): 146-152.

5 - Wei LA, Chen HH, Hink EM, Durairaj VD. Pediatric facial fractures from dog bites. Ophthalmic Plast Reconstr Surg. 2013; 29(3): 179-82. doi: 10.1097/IOP.0b013e3182880114.

6 - Richardson S, Sharma K, Khandeparker RV. Management of human bite injury of the upper and lower eyelids: a rare case report. J Korean Assoc Oral Maxillofac Surg. 2016; 42(6): 375-378. doi: 10.5125/ jkaoms.2016.42.6.375.

7 - Chang EI, Esmaeli B, Butler CE. Eyelid Reconstruction. Plast Reconstr Surg. 2017; 140(5): 724e-735e. doi: 10.1097/PRS.0000000000003820.

8 - Agrawal A, Kumar P, Singhal R, Singh V, Bhagol A. Animal Bite Injuries in Children: review of literature and case series. Int J Clin Pediatr Dent. 2017; 10(1): 67-72. doi: 10.5005/jp-journals-10005-1410. 
9- Smith D. Dog bites in children: an oculoplastic urgency in ER and OR. EyeNet. 2013; 41-3.

10 - Macedo JL, Rosa SC, Queiroz MN, Gomes TG. Reconstruction of face and scalp after dog bites in children. Rev Col Bras Cir. 2016; 43(6): 452457. doi: 10.1590/0100-69912016006007.

11 - Alghoul M, Pacella SJ, McClellan WT, Codner MA. Eyelid reconstruction. Plast Reconstr Surg. 2013; 132(2): 288e-302e. doi: 10.1097/PRS.0b013e3182958e6b.

12 - Alghoul MS, Bricker JT, Vaca EE, Purnell CA. Lower eyelid reconstruction: a new classification incorporating the vertical dimension. Plast Reconstr Surg. 2019; 144(2): 443-455. doi: 10.1097/ PRS.00000000000005882.

13 - Morley AM, Sousa JL, Selva D, Malhotra R. Techniques of upper eyelid reconstruction. Surv Ophthalmol. 2010; 55(3): 256-71. doi: 10.1016/j. survophthal.2009.10.004.

14 - Rajak SN, Malhotra R, Selva D. The 'over-the-top' modified CutlerBeard procedure for complete upper eyelid defect reconstruction. Orbit. 2019; 38(2): 133-136. doi: 10.1080/01676830.2018.1444061.

15 - Herford AS, Cicciu M, Clark A. Traumatic eyelid defects: a review of reconstructive options. J Oral Maxillofac Surg. 2009; 67(1): 3-9. doi: 10.1016/j.joms.2008.09.009.

16 - Lee WW, Erickson BP, Ko MJ, Liao SD, Neff A. Advanced single-stage eyelid reconstruction: anatomy and techniques. Dermatol Surg. 2014; 40 (Suppl 9): S103-12. doi: 10.1097/DSS.0000000000000117.

17 - Poh EW, O’Donnell BA, McNab AA, Sullivan TJ, Gaskin B, Malhotra R, et al. Outcomes of upper eyelid reconstruction. Ophthalmology. 2014; 121(2): 612-3.e1. doi: 10.1016/j.ophtha.2013.10.010. Epub 2013 Nov 21.

18 - Gibreel W, Harvey JA, Garrity J, Bite U. Lower Eyelid Reconstruction Using a Nasolabial, Perforator-based V-Y Advancement Flap: Expanding the Utility of Facial Perforator Flaps. Ann Plast Surg. 2019 Jan; 82(1): 46-52. doi: 10.1097/SAP.00000000000001576.

19 - Ehmke M, Schwipper V. Surgical reconstruction of eyelids. Facial Plast Surg. 2011; 27(3): 276-83. doi: 10.1055/s-0031-1275777.

20 - Stein JD, Antonyshyn OM. Aesthetic eyelid reconstruction. Clin Plast Surg. 2009; 36(3): 379-97. doi: 10.1016/j.cps.2009.02.011.

21 - Wu PS, Beres A, Tashjian DB, Moriarty KP. Primary repair of facial dog bite injuries in children. Pediatr Emerg Care. 2011; 27(9): 801-3. doi: 10.1097/PEC.0b013e31822c1112.

22 - Imran D, Mandal A. A dog bite to the eyelid. J R Soc Med. 2004; 97(2): 78-9. doi: 10.1258/jrsm.97.2.78. 
23 - Bourguignon-Filho AM, Feitosa ACR, Beltrão GC, Pagnoncelli RM. Utilização do laser de baixa intensidade no processo de cicatrização tecidual: revisão de literatura. Rev Port Estomatol Cir Maxilofac. 2005; 46(1): 37-43.

24 - Karimnejad K, Walen S. Complications in Eyelid Surgery. Facial Plast Surg Clin North Am. 2016; 24(2): 193-203. doi: 10.1016/j.fsc.2015.12.008. PMID: 27105805.

25 - Brasil. Ministério da Saúde. Nota Informativa nº 26-SEI/2017-CGPNI/ DEVIT/SVS/MS. Brasília: Ministério da Saúde; 2017. 


\title{
Aesthetic-functional reconstruction of upper and lower eyelids in a pediatric patient after a canine attack: a case report
}

\begin{abstract}
Canine attacks can cause serious injuries and disfigurements in the face of children, to the point that they need specialized and urgent care. The involvement of the periocular region requires a thorough assessment to identify possible bone fractures, eyelid injuries, eye and lacrimal system injuries, along with ophthalmic follow-up. This article aims to report the case of a 07-year-old child, victim of a dog bite in the face. The attack resulted in partial avulsion of upper and lower eyelids of the right eye. After performing the eyelid reconstruction procedure and anti-rabies prophylactic care, the patient progresses with satisfactory esthetic-functional reestablishment and without signs and symptoms of rabies disease. This report emphasizes the importance of adequate management of injuries caused by a canine attack in the eyelid region, in a pediatric patient.
\end{abstract}

KEYWORDS: Bites and Stings; Dogs; Eyelids; Oral and Maxillofacial Surgeons; Wounds and Injuries

\section{Como citar este artigo}

Paula LM, Prata Júnior AR, Oliveira MTF. Reconstrução estético-funcional das pálpebras superior e inferior em paciente pediátrico após ataque canino: relato de caso. Rev Odontol Bras Central 2021; 30(89): 422-433. DOI: 10.36065/robrac.v30i89.1519 